\title{
On confidence intervals from micropalaeontological counts
}

\author{
STEFAN A. REVETS \\ Koninklijk Belgisch Instituut voor Natuurwetenschappen, Departement Paleontologie, Vautierstraat 29, B-1000, Brussel, Belgium \\ (e-mail: s.revets@tiscali.be).
}

\begin{abstract}
In micropalaeontology, statistics resulting from counts are rarely reported with error margins or confidence intervals. A short discussion of taxon proportions and of Fisher's $\alpha$ diversity index is augmented with tables and graphs to assist the researcher in calculating confidence intervals. Any results, discussions or further calculations based on counts can then be put on a more secure statistical footing. J. Micropalaeontol. 23(1): 61-65, May 2004.
\end{abstract}

\section{INTRODUCTION}

Quantitative studies in micropalaeontology continue to develop, with new and powerful multivariate statistical methods being tried and adopted. However, confidence intervals are rarely, if ever, calculated, let alone referred to. All too often, numbers from counts are presented as being both accurate and precise and used as such in more complex methods. This omission is detrimental as it makes it very difficult to gauge significance and reliability of any outcome of such statistical operations.

Although the statistics of sampling are very well established, tables and graphs of immediate use to the micropalaeontologist are not as readily available as one might wish. This article deals only with the numbers obtained from the picking tray or slide traverse. The problems of dealing adequately with the sampling of a population as it occurs out there in the world are different from the problems of correctly measuring and representing the taxa present in the physical samples taken. Therefore, the term 'population' is used here in the statistical sense, referring to the specimens in the collected rock sample or in the (washed) residue, not to the biological population out in the field.

It is relevant and important to have a reliable and correct measure of the precision with which one counts and calculates taxon presence and proportions. When working with extant organisms and their heterogeneous distribution, the measure of 'laboratory precision' has a direct bearing on the nature and, particularly, the intensity of any sampling program in the field. In marked contrast, many, if not most, micropalaeontologists work with fossil material from outcrop or drillcore. They simply cannot afford the luxury of dealing with such ecological considerations and issues of replicate sampling. Nevertheless, palaeoecological interpretations have to be made from whatever material could be recovered. Such interpretations necessarily rely on the abundances of any taxa encountered and statistically justified confidence intervals are, therefore, important. Anyone confronted with such problems will hopefully benefit from the following discussion, tables and graphs.

\section{CONFIDENCE INTERVALS ON PROPORTIONS}

A general problem which has greatly exercised the minds of researchers and statisticians alike is the minimal size of a sample required to answer a particular question. The micropalaeontologist, in particular, is confronted with the question of how many, or few, specimens to pick from a rock sample or washed residue and still retain confidence in the detection level and possibly proportions calculated of the taxa in the sample. Phleger (1960) applied the results obtained by Dryden (1931) and proposed the now commonly used 300 specimens as a sufficient and practical number with which to determine relative abundances of foraminiferal species. In a different context, Shaw (1964) also used the Binomial Distribution and arrived at the same kind of answers. Dennison \& Hay (1967) applied Shaw's analysis to the problem of the size of sample area and provided a very useful graph (calculated and refigured here in Fig. 1). They also discussed the wider applicability of their results, including amongst others Dryden's and Phleger's work.

Their plot shows the number of specimens required if one is prepared to overlook with a probability $P$ a species making up a particular proportion in the population. For example, the graph in Figure 1 shows that if one is prepared to miss out 1 in 20 $(p=0.05)$ species which make up a proportion of 0.01 of the population, then one needs to count 300 specimens.

What these analyses and associated graphs do not provide is a measure of the precision of these proportions. There is a substantial difference between finding a taxon making up a particular proportion in a sample and calculating that proportion. Many, if not most, taxa are rare (Fisher et al., 1943; Buzas et al., 1982) and may well play an important role in ecology, palaeoecology and any assessment or characterization of environments (Cao et al., 1998; Cao \& Larsen, 2001). It is, therefore, important and relevant to be able to justify statistically any measurement of occurrence of such rare taxa.

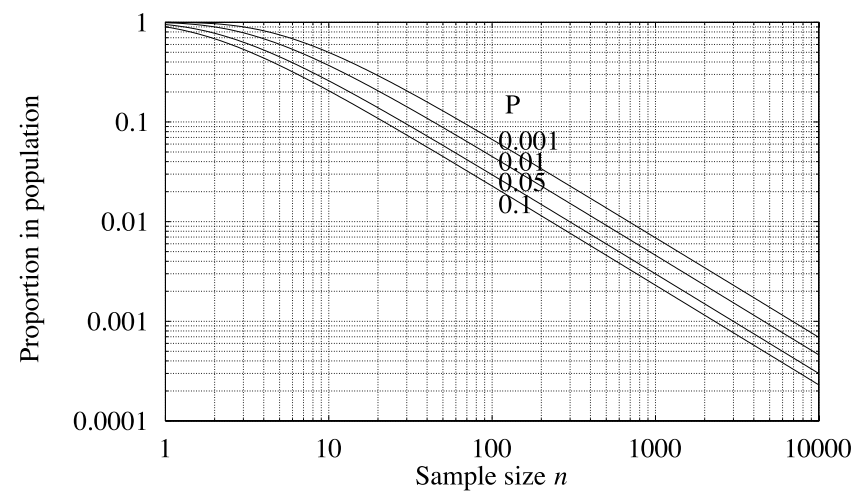

Fig. 1. Estimating sample size. For a number of probabilities of failing to find a taxon, the curves show how many specimens must be counted to detect a taxon present at a particular proportion in the population samples. 
Finding specimens of rare species in a sample is a good example of a discontinuous stochastic process known as the Poisson Process - well described by the Poisson Distribution (Poisson, 1837; Student, 1907). There are benefits in deriving this distribution from discontinuous stochastic processes (e.g. Hald, 1952). A very important, but all too often overlooked, result is that the Poisson Distribution describes a process in its own right and should not be seen as a limiting case of the Binomial Distribution.

Thanks to the properties of the Poisson Distribution (some of which are discussed in the Appendix), confidence intervals on any observed number of occurrences can be derived and calculated. The value and the power of using a Poisson Distribution lies in the fact that it allows one to deal directly with the number of occurrences. This is in marked contrast to the Binomial Distribution which needs both the number of occurrences and the total number of events.

With the Poisson Distribution, it is a straightforward matter to derive the confidence limits (equations 4 ) and then calculate the confidence interval on the proportion of the species of interest. Rather than have the reader refer to $\chi^{2}$ tables to calculate confidence intervals, Table 1 lists the lower and upper limits of the $0.99,0.95$ and 0.90 confidence intervals for number of specimens between 0 and 50 .

An example will clarify the use of this table. Suppose that six specimens of a taxon are found in a picking tray. From Table 1 one reads that the 0.95 confidence interval for six specimens lies between 2.2 and 13.1. The proportion and the 0.95 confidence limits of this proportion are simply these numbers divided by the total number of specimens counted or picked from the tray. If 100 specimens were found in total, the species would make up 0.06 on average of the population and this proportion would lie anywhere between 0.02 and 0.13 . Similarly, if 300 specimens had yielded the six specimens, the proportion would be 0.02 with the 0.95 confidence interval stretching from 0.007 to 0.044 .

Inspection of the table shows the asymmetric nature of the confidence intervals, especially for the smaller values. This asymmetry is a clear indication of the non-Gaussian nature of the distribution and argues against the use of normal approximations (Garwood, 1936; Hald, 1952). With the table calculated here, there is no need to resort to approximations: the values presented are exact.

A related, but different, problem is posed if one wishes to know if two taxa make up different proportions in the population. How much of a difference in counts is necessary for one to conclude that the taxa make up different proportions? Or indeed, how much of a difference in counts may one allow for the taxa to occur in the same proportions?

Once again, thanks to the properties of the Poisson Distribution, these critical numbers can be calculated exactly. The calculations are straightforward but tedious (see equation 9), therefore, the results are listed here in Table 2. The table shows for counts from 0 to 50 of a taxon A how many specimens of a taxon $\mathrm{B}$ have to be found for $\mathrm{B}$ to be deemed more abundant than A, at the usual confidence levels of $0.90,0.95$ and 0.99 .

Revisiting the previous example with six specimens of species A, how many specimens does one need of species B before it can be said to be more abundant (and make up a larger proportion

\begin{tabular}{|c|c|c|c|c|c|c|}
\hline \multirow{2}{*}{$\frac{n}{0}$} & \multicolumn{2}{|c|}{0.99} & \multicolumn{2}{|c|}{0.95} & \multicolumn{2}{|c|}{0.90} \\
\hline & 0.0000 & 5.2983 & 0.0000 & 3.6888 & 0.0000 & 2.9957 \\
\hline 1 & 0.0050 & 7.4301 & 0.0253 & 5.5716 & 0.0512 & 4.7438 \\
\hline 2 & 0.1034 & 9.2737 & 0.2422 & 7.2246 & 0.3553 & 6.2957 \\
\hline 3 & 0.3378 & 10.9774 & 0.6186 & 8.7672 & 0.8176 & 7.7536 \\
\hline 4 & 0.6722 & 12.5940 & 1.0898 & 10.2415 & 1.3663 & 9.1535 \\
\hline 5 & 1.0779 & 14.1497 & 1.6234 & 11.6683 & 1.9701 & 10.5130 \\
\hline 6 & 1.5369 & 15.6596 & 2.2018 & 13.0594 & 2.6130 & 11.8424 \\
\hline 7 & 2.0373 & & 2.8143 & 14.4226 & 3.2853 & 1481 \\
\hline 8 & 2.5711 & 18.5782 & 3.4538 & 15.7631 & 3.9808 & 14.4346 \\
\hline 9 & 3.1324 & 19.9984 & 4.1153 & 17.0848 & 4.6952 & 15.7052 \\
\hline 10 & 3.7169 & 21.3978 & 4.7953 & 18.3903 & 5.4254 & 16.9622 \\
\hline 11 & 4.3213 & 22.7792 & 5.4911 & 19.6820 & 6.1690 & 18.2075 \\
\hline 12 & 4.9431 & 24.1449 & 6.2005 & 20.9615 & 6.9242 & 19.4425 \\
\hline 13 & 5.5801 & 25.4966 & 6.9219 & 22.2304 & 7.6895 & 20.6685 \\
\hline 14 & 6.2306 & 26.8359 & 7.6539 & 23.4896 & 8.4639 & 21.8864 \\
\hline 15 & 6.8933 & 28.1640 & 8.3953 & 24.7402 & 9.2463 & 23.0971 \\
\hline 16 & 7.5670 & 29.4819 & 9.1453 & 25.9830 & 10.0359 & 24.3011 \\
\hline 17 & 8.2506 & 30.7905 & 9.9031 & 27.2186 & 10.8321 & 25.4992 \\
\hline 18 & 8.9433 & 32.0907 & 10.6679 & 28.4477 & 11.6343 & 26.6917 \\
\hline 19 & 9.6444 & 33.3829 & 11.4392 & 29.6708 & 12.4419 & 27.8792 \\
\hline 20 & 10.3532 & 34.6680 & 12.2165 & 30.8883 & 13.2546 & 29.0620 \\
\hline 21 & 11.0692 & 35.9462 & 12.9993 & 32.1007 & 14.0720 & 30.2404 \\
\hline 22 & 11.7918 & 37.2182 & 13.7872 & 33.3082 & 14.8937 & 31.4148 \\
\hline 23 & 12.5206 & 38.4843 & 14.5800 & 34.5112 & 15.7195 & 32.5853 \\
\hline 24 & 13.2553 & 39.7449 & 15.3772 & 35.7101 & 16.5490 & 33.7524 \\
\hline 25 & 13.9953 & 41. & 86 & 3 & 321 & 160 \\
\hline 26 & 14.7405 & 42.2 & 16.9 & 38. & 18. & 36.0766 \\
\hline 27 & 15.4906 & 43.4 & 17.7931 & 39.2 & 19.0581 & 37.2341 \\
\hline 28 & 16.2452 & 44.7384 & 18.6058 & 40.4678 & 19.9006 & 38.3889 \\
\hline 29 & 17.0041 & 45.9758 & 19.4217 & 41.6488 & 20.7459 & 39.5409 \\
\hline 30 & 17.7672 & 47.2093 & 20.2408 & 42.8268 & 21.5939 & 40.6905 \\
\hline 31 & 18.5342 & 48.4390 & 21.0630 & 44.0020 & 22.4445 & 41.8376 \\
\hline 32 & 19.3048 & 49.6652 & 21.8879 & 45.1744 & 23.2974 & 42.9824 \\
\hline 33 & 20.0791 & 50.88 & 22.7156 & 46.3442 & 24.1526 & 44.1250 \\
\hline 34 & 20.8567 & 52.1074 & 23.5459 & 47.5115 & 25.0101 & 45.2656 \\
\hline 35 & 21.6375 & 53.3238 & 24.3787 & 48.6765 & 25.8696 & 46.4041 \\
\hline 36 & 22.4215 & 54.5371 & 25.2139 & 49.8391 & 26.7311 & 47.5407 \\
\hline 37 & 23.2084 & 55.7476 & 26.0514 & 50.9996 & 27.5946 & 48.6754 \\
\hline 38 & 23.9982 & 56.9554 & 26.8910 & 52.1579 & 28.4599 & 49.8084 \\
\hline 39 & 24.7908 & 58.1605 & 27.7328 & 53.3142 & 29.3269 & 50.9397 \\
\hline 40 & 25.5859 & 59.3630 & 28.5765 & 54.4686 & 30.1957 & 52.0693 \\
\hline 41 & 26.3836 & 60.5631 & 29.4223 & 55.6211 & 31.0661 & 53.1974 \\
\hline 42 & 27.1838 & 61.7608 & 30.2699 & 56.7718 & 31.9381 & 54.3239 \\
\hline 43 & 27.9863 & 62.9562 & 31.1193 & 57.9207 & 32.8116 & 55.4490 \\
\hline 44 & 28.7911 & 64.1494 & 31.9704 & 59.0679 & 33.6866 & 56.5726 \\
\hline 45 & 29.5981 & 65.3405 & 32.8233 & 60.2135 & 34.5630 & 57.6948 \\
\hline 46 & 30.4072 & 66.5295 & 33.6777 & 61.3575 & 35.4407 & 58.8158 \\
\hline 47 & 31.2184 & 67.7165 & 34.5338 & 62.5000 & 36.3198 & 59.9354 \\
\hline 48 & 32.0316 & 68.9015 & 35.3914 & 63.6410 & 37.2002 & 61.0538 \\
\hline 49 & 32.8467 & 70.0847 & 36.2504 & 64.7806 & 38.0819 & 62.1710 \\
\hline 50 & 33.6637 & 71.2660 & 37.1109 & 65.9187 & 38.9647 & 63.2870 \\
\hline
\end{tabular}

Table 1. Confidence intervals for the Poisson Distribution. The list shows for an observed number of occurrences $n$ the lower and upper limits at probabilities $0.99,0.95$ and 0.90 .

of the population)? Looking under the entries for six in Table 2 shows that at least 16 specimens of species $\mathrm{B}$ are required (at a probability of 0.95 ). These numbers can then be translated to proportions by dividing them by the total number of specimens counted.

If, amongst the counts, there are 10 specimens of species $\mathrm{A}$ and 20 of species $\mathrm{B}$, one looks up the entry for 10 to find that at least 21 specimens are needed for B to be more abundant (at 0.95 


\begin{tabular}{|c|c|c|c|}
\hline$n$ & 0.99 & 0.95 & 0.90 \\
\hline 0 & 8 & 5 & 4 \\
\hline 1 & 11 & 7 & 6 \\
\hline 2 & 12 & 9 & 7 \\
\hline 3 & 14 & 10 & 9 \\
\hline 4 & 16 & 12 & 10 \\
\hline 5 & 17 & 13 & 12 \\
\hline 6 & 19 & 15 & 13 \\
\hline 7 & 21 & 16 & 14 \\
\hline 8 & 22 & 18 & 16 \\
\hline 9 & 24 & 19 & 17 \\
\hline 10 & 25 & 21 & 18 \\
\hline 11 & 27 & 22 & 20 \\
\hline 12 & 28 & 23 & 21 \\
\hline 13 & 30 & 25 & 22 \\
\hline 14 & 31 & 26 & 23 \\
\hline 15 & 32 & 27 & 25 \\
\hline 16 & 34 & 28 & 26 \\
\hline 17 & 35 & 30 & 27 \\
\hline 18 & 37 & 31 & 28 \\
\hline 19 & 38 & 32 & 29 \\
\hline 20 & 39 & 33 & 31 \\
\hline 21 & 41 & 35 & 32 \\
\hline 22 & 42 & 36 & 33 \\
\hline 23 & 43 & 37 & 34 \\
\hline 24 & 45 & 38 & 35 \\
\hline 25 & 46 & 40 & 37 \\
\hline 26 & 47 & 41 & 38 \\
\hline 27 & 49 & 42 & 39 \\
\hline 28 & 50 & 43 & 40 \\
\hline 29 & 51 & 45 & 41 \\
\hline 30 & 53 & 46 & 42 \\
\hline 31 & 54 & 47 & 44 \\
\hline 32 & 55 & 48 & 45 \\
\hline 33 & 56 & 49 & 46 \\
\hline 34 & 58 & 51 & 47 \\
\hline 35 & 59 & 52 & 48 \\
\hline 36 & 60 & 53 & 49 \\
\hline 37 & 62 & 54 & 51 \\
\hline 38 & 63 & 55 & 52 \\
\hline 39 & 64 & 57 & 53 \\
\hline 40 & 66 & 58 & 54 \\
\hline 41 & 67 & 59 & 55 \\
\hline 42 & 68 & 60 & 56 \\
\hline 43 & 69 & 61 & 57 \\
\hline 44 & 70 & 62 & 58 \\
\hline 45 & 72 & 64 & 60 \\
\hline 46 & 73 & 65 & 61 \\
\hline 47 & 74 & 66 & 62 \\
\hline 48 & 75 & 67 & 63 \\
\hline 49 & 76 & 68 & 64 \\
\hline 50 & 77 & 69 & 65 \\
\hline
\end{tabular}

Table 2. Critical values for the discrimination of two Poisson-distributed samples. Given a number of specimens $n$ for one species, the list shows the minimal number required for another species to occur with a higher abundance, at confidence probabilities of $0.99,0.95$ and 0.90 .

probability): therefore, one has to conclude that both taxa occur with the same abundance.

From Table 2 it can also be seen that a count of 300 specimens is just sufficient to differentiate at the 0.95 confidence level between a 0.01 and 0.04 proportion in a population: a count of three differs significantly from 12 (at 0.95 confidence

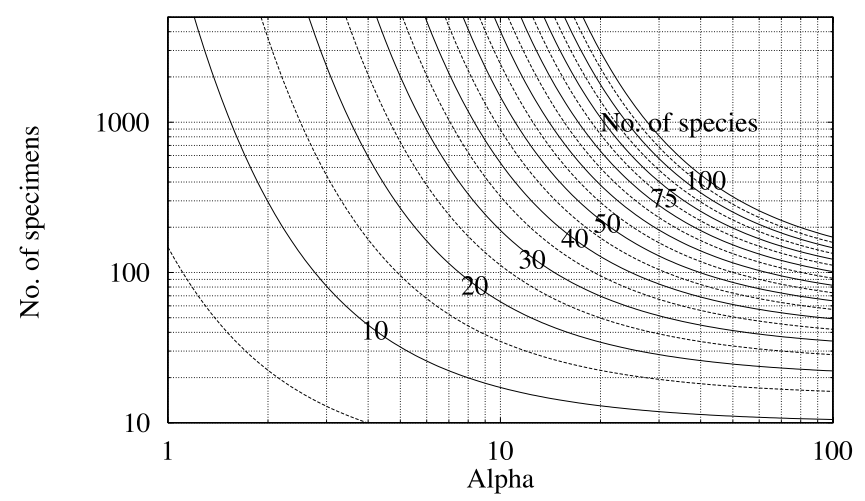

Fig. 2. Fisher's $\alpha$ diversity index. From the number of specimens laid out on the $y$-axis the $\alpha$ index can be read off on the $x$-axis using the curve with the number of species found. The successive curves increase with a step of five species from 5 to 100 .

level), which, divided by 300 , translates into differentiating a $1 \%$ from a $4 \%$ proportion.

\section{CONFIDENCE INTERVALS ON FISHER'S ALPHA INDEX}

This diversity index was proposed by Fisher as a natural extension of the Poisson Distribution (Fisher et al., 1943). In a great many cases, the distribution of species in function of the numbers of specimens found can be described as a negative binomial distribution, i.e. many taxa are represented by only a few specimens while only a very few occur in great numbers. The $a$ index characterizes with a single number the shape of this distribution: it gives the number of species represented by a single specimen in a sample, whatever its size. Unfortunately, the index is an awkward function of two variables - number of specimens and number of species - and direct calculation is not possible. Various kinds of graphs can be made up to show the relation: Figure 2 is such a graph, which differs in portrayal from the one used most often in micropalaeontological circles since its introduction and excellent discussion by Murray (1968, 1973).

The nature of the index is counterintuitive: it seems odd that the number of taxa represented by a single specimen should remain constant, regardless of the number of specimens collected. In practice, an increase in the value of the index is often encountered. In the original article by Fisher et al., Williams showed increases in $a$ (from 31.38 to 40.24 ) when continuing to collect lepidoptera over a four-year period - and that with specimen numbers running up to 15000 . Murray (1968) also found an increase in $a$ when going on picking foraminifera from 100 to about 600 specimens. Nevertheless, he concluded that the variation he had come across was not sufficiently large to cause concern.

A rerun of Murray's counting experiment on two different samples failed to reproduce this increase. The samples used were the Sands of Wemmel, Belgium (Eocene, Lutetian) and from Antikephalina Bay, Paros, Greece (Recent). The samples had been washed on a $63 \mu \mathrm{m}$ sieve and portions randomly scattered on a picking tray. Table 3 shows the results: these should allay any concern about the validity of the $\alpha$ index as a consistent measure of diversity. 


\begin{tabular}{lccccc}
\hline \multicolumn{3}{c}{ Wemmel Sands, Belgium } & \multicolumn{3}{c}{ Paros, Greece } \\
$N$ & $S$ & $\alpha$ & $N$ & $S$ & $\alpha$ \\
\hline 142 & 29 & $11.0 \pm 3.0$ & 192 & 45 & $18.6 \pm 4.0$ \\
228 & 32 & $10.5 \pm 2.5$ & 344 & 52 & $16.5 \pm 3.0$ \\
353 & 37 & $10.5 \pm 2.0$ & 409 & 58 & $18.5 \pm 2.9$ \\
556 & 42 & $10.6 \pm 1.9$ & 502 & 62 & $18.6 \pm 2.9$ \\
757 & 42 & $9.6 \pm 1.5$ & 620 & 70 & $20.2 \pm 2.8$ \\
\hline
\end{tabular}

Table 3. Cumulative counts of specimens $(N)$ and species $(S)$ with the resulting Fisher's $\alpha$ index from a sample of the Eocene Sands of Wemmel and a sample of the Recent of Paros.

When Fisher proposed this index, he also investigated the effects of variation. His calculation of the variance of the index is of particular interest. As one would expect from the functions defining the index, the formula for the variance is somewhat involved and its relation to the parameters making it up number of species and number of specimens - is far from transparent. However, by adopting the coefficient of variation (standard deviation divided by the mean), a highly informative graph can be drawn by plotting the coefficient of variation against the number of specimens used for a selection of values of $a$, as shown in Figure 3.

It transpires from this graph that the coefficient of variation at counts of 300 specimens is about 0.1 , or, that the standard deviation amounts to about one-tenth $(0.092-0.145$ to be exact) of $a$, and that for a very wide range of values of $a(100-2)$. This means that the $95 \%$ confidence interval of $a$ when calculated from 300 counted specimens, is about $20 \%$ of its value (i.e. $0.1 \times 1.96$ which is near enough 0.2 , or $20 \%$ ), a substantial amount. It takes roughly an order of magnitude more in specimens to be counted, about 3000 , to reduce this confidence interval to plus or minus $10 \%$ of the value of $a$. These surprisingly large intervals should be borne in mind when attempting to compare and contrast samples.

\section{MATHEMATICS APPENDIX}

\section{The Poisson Distribution}

The Poisson Distribution was proposed by Poisson (1837) and independently by 'Student' (pseudonym of W. S. Gosset, 1907). It is a function defined by a single parameter $m$. This remarkable and valuable property contributes substantially to the power of

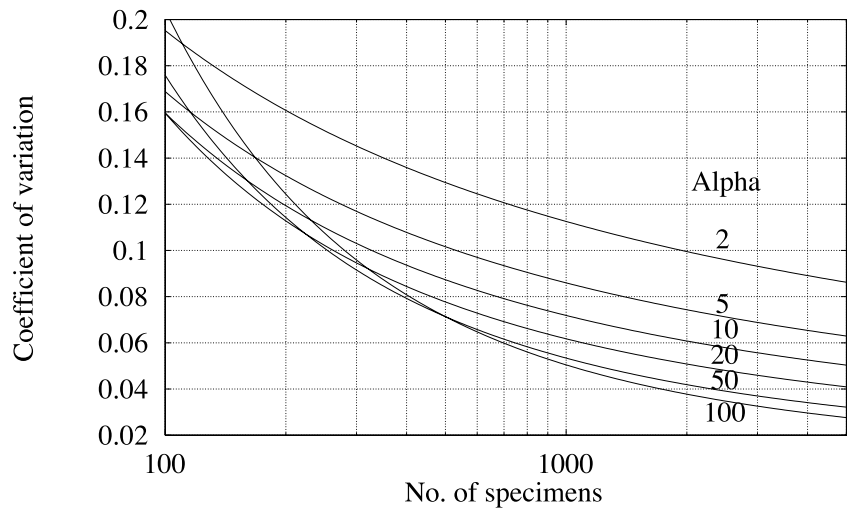

Fig. 3. Variation coefficient of Fisher's $\alpha$ diversity index. A plot of the coefficient of variation of $\alpha$ in function of the number of specimens used to calculate $\alpha$, and that for a selection of values of $\alpha$. this function in many of its applications. In contrast, all other distributions are functions determined by two or more parameters. The probability distribution is defined as

$$
p(x)=e^{-m} \frac{m^{x}}{x !}, \quad x=0,1,2 \ldots
$$

Confidence intervals. The cumulative distribution function can be shown to be related to the $\chi^{2}$ distribution (Deming, 1950; Hald, 1952):

$$
P(x)=1-P\left(\chi^{2}<2 m\right), \quad f=2(x+1)
$$

This relation gives the means with which to calculate confidence intervals for the parameter $m$ of the Poisson Distribution:

$$
P\left\{\underline{\mathrm{m}}\left(x_{0}\right) \leq m \leq \overline{\mathrm{m}}\left(x_{0}\right)\right\}=P_{2}-P_{1}
$$

yielding

$$
\begin{cases}\overline{\mathrm{m}}=\frac{1}{2} \chi_{\left(1-P_{1}\right)}^{2}, & f=2\left(x_{0}+1\right) \\ \underline{\mathrm{m}}=\frac{1}{2} \chi_{\left(1-P_{2}\right)}^{2}, & f=2 x_{0}\end{cases}
$$

Since the $\chi^{2}$ distribution is defined through a $\Gamma$-function, the values of the confidence intervals can be calculated directly. Table 1 lists the results of such calculations carried out with the Mathematica program.

Critical values. To find out if two observations come from two Poisson Distributions with the same or with different means relies on the addition theorem for the Poisson Distribution. This easily proved theorem states that if $x_{1}, x_{2}, \ldots, x_{n}$ are stochastically independent and Poisson-distributed with means $m_{1}, m_{2}$, $\ldots, m_{n}$ then the sum $x=x_{1}+x_{2}+\ldots+x_{n}$ will be Poissondistributed with mean $m=m_{1}+m_{2}+\ldots+m_{n}$.

With the null hypothesis that the two means are equal, the probability of observing $n_{1}, n_{2}$ is simply

$$
p\left(n_{1}+n_{2}\right)=e^{-2 m} \frac{(2 m)^{n_{1}+n_{2}}}{\left(n_{1}+n_{2}\right) !}
$$

and, therefore, the conditional probability becomes

$$
p\left(n_{1} \mid n_{1}+n_{2}\right)=\frac{p\left(n_{1}, n_{2}\right)}{p\left(n_{1}+n_{2}\right)}=\frac{\left(n_{1}+n_{2}\right) !}{n_{1} ! n_{2} !}\left(\frac{1}{2}\right)^{n_{1}+n_{2}}
$$

To test the hypothesis that $m_{1}=m_{2}$ against the alternative of $m_{1}>m_{2}$, one requires the probability

$$
\begin{aligned}
& P\left(n \geq n_{1} \mid n_{1}+n_{2}\right)=\left(\frac{1}{2}\right)^{n_{1}+n_{2}} \sum_{n=n_{1}}^{n_{1}+n_{2}}\left(\begin{array}{c}
n_{1}+n_{2} \\
n
\end{array}\right) \\
= & 1-P\left(F<\frac{n_{1}}{n_{2}+1}\right), \quad f_{1}=2\left(n_{2}+1\right), f_{2}=2 n_{1}
\end{aligned}
$$


If this probability is less than or equal to the significance level $\alpha$, then reject the null hypothesis that $m_{1}=m_{2}$. Alternatively, the equation can be transformed to

$$
\frac{n_{1}}{n_{2}+1} \geq F_{1-a}\left(2\left(n_{2}+1\right), 2 n_{1}\right)
$$

which allows for the calculation from the F-Distribution of the critical values for which the means of the parent Poisson Distributions differ. The critical values at significance levels $0.99,0.95$ and 0.90 are listed for $n_{2}$ values between 0 and 50 in Table 2.

\section{Fisher's $\alpha$}

Fisher derived the $\alpha$ index by extending the Poisson Distribution by considering the effects of the parameter (the mean) having a distribution of its own. He chose the Euler function (now usually known as the $\mathrm{T}$-function), and arrived at the expression

$$
f(n)=\frac{(k+n-1) !}{n !(k-1) !} \frac{p^{n}}{(1+p)^{k+n}}
$$

and setting the constant factor in the denominator $(k-1) !=a$, $k=0$ and $p /(p+1)=x$, simplified it to

$$
\frac{a}{n} x^{n}
$$

Through summations, Fisher calculated the expected number of species $S$ and the number of individuals $N$ to obtain

$$
S=-a \ln (1-x), \quad N=\frac{a x}{1-x}
$$

By eliminating $x$ from these two formulae the equation becomes the well-known

$$
S=a \ln \left(1+\frac{N}{a}\right)
$$

Fisher also derived a formula for the variance of $\alpha$

$$
s_{a}^{2}=\frac{a^{3}\left\{(N+a)^{2} \ln \left(\frac{2 N+a}{N+a}\right)-a N\right\}}{(S N+S a-a N)^{2}}
$$

The coefficient of variation is defined as the ratio between standard deviation and mean: applying this to the variance formula for $\alpha$ yields

$$
c_{a}=\frac{\sqrt{a\left\{(N+a)^{2} \ln \left(\frac{2 N+a}{N+a}\right)-a N\right\}}}{S N+S a-a N}
$$

\section{Manuscript received 7 April 2003 \\ Manuscript accepted 4 December 2003}

\section{REFERENCES}

Buzas, M.A., Koch, S.J., Culver, S.J. \& Sohl, N.F. 1982. On the distribution of species occurrence. Paleobiology, 8: 142-150.

Cao, Y. \& Larsen, D.P. 2001. Rare species in multivariate analysis for bioassessment: some considerations. Journal of the North American Benthological Society, 20: 144-153.

Cao, Y., Williams, D.D. \& Williams, N.E. 1998. How important are rare species in aquatic community ecology and bioassessment? Limnology and Oceanography, 43: 1403-1409.

Deming, W.E. 1950. Some Theory of Sampling. Dover Publications, New York.

Dennison, J.M. \& Hay, W.W. 1967. Estimating the needed sampling area for subaquatic ecological studies. Journal of Paleontology, 41: 706-708.

Dryden, A.L. 1931. Accuracy in percentage representations of heavy mineral frequencies. Proceedings of the National Academy of Sciences, 17: $233-238$.

Fisher, R.A., Corbet, A.S. \& Williams, C.B. 1943. The relation between the number of species and the number of individuals in a random sample of an animal population. Journal of Animal Ecology, 12: $42-57$

Garwood, F. 1936. Fiducial limits for the Poisson Distribution. Biometrika, 28: 437-442.

Hald, A. 1952. Statistical Theory with Engineering Applications. Wiley \& Sons, New York

Murray, J.W. 1968. Living foraminifers of lagoons and estuaries. Micropaleontology, 14: 83-96.

Murray, J.W. 1973. Distribution and ecology of living benthic foraminiferids. Heinemann, London.

Phleger, F.B. 1960. Ecology and distribution of Recent Foraminifera. John Hopkins University Press, Baltimore.

Poisson, S.D. 1837. Recherches sur la probabilité des jugements en matière criminelle et en matière civile, precedées des règles générales du calcul des probabilités, Paris.

Shaw, A.E. 1964. Time in Stratigraphy. McGraw-Hill, New York.

Student 1907. On the error of counting with a haemacytometer. Biometrika, 5: 351-360. 